\title{
Modulated wavepackets associated with longitudinal dust grain oscillations in a dusty plasma crystal *
}

\author{
I. Kourakis \\ Institut für Theoretische Physik IV, Fakultät für Physik und Astronomie, \\ Ruhr-Universität Bochum, D-44780 Bochum, Germany \\ (Dated: Received 5 December 2003; accepted 13 January 2004)
}

\begin{abstract}
The nonlinear amplitude modulation of longitudinal dust lattice waves (LDLWs) propagating in a dusty plasma crystal is investigated in a continuum approximation. It is shown that long wavelength LDLWs are modulationally stable, while shorter wavelengths may be unstable. The possibility for the formation and propagation of different envelope localized excitations is discussed. It is shown that the total grain displacement bears a (weak) constant displacement (zeroth harmonic mode), due to the asymmetric form of the nonlinear interaction potential. The existence of asymmetric envelope localized modes is predicted. The types and characteristics of these coherent nonlinear structures are discussed.

PACS numbers: 52.27.Lw, 52.35.Fp, 52.25.Vy
\end{abstract}

\section{INTRODUCTION}

A remarkable new feature associated with the physics of dust-contaminated plasmas [1, 2] is the existence of strongly-coupled charged matter configurations, which even lead to the formation of dusty plasma (DP) Wigner-type layers (crystals) when the inter-grain potential energy far exceeds the average kinetic energy of the dust particles. Dust crystals display a variety of new phenomena, such as phase transitions (crystallization, melting) and lattice excitations; a link is thus established between plasma physics and solid state physics [3].

Dust lattices, which are typically formed in the sheath region in discharge experiments and remain suspended above the negative electrode due to a balance between the external electric and gravity forces [4], are known to support harmonic oscillations (acoustic modes) in both longitudinal and transverse-shear (horizontal-plane) directions, as well as optical-mode-like oscillations in the vertical (off-plane) direction [5, 6, 7, 8, 9, 10].

Dust-lattice waves (DLWs) are reminiscent of waves ('phonons') propagating in atomic chains, which are long known to be dominated by interesting nonlinear phenomena (localized modes, instabilities), due to the intrinsic nonlinearities of inter-atomic interaction mechanisms and/or on-site substrate potentials 11, 12, 13, 14]. The present study is devoted to the study of one such phenomenon, namely the nonlinear amplitude modulation of weakly nonlinear oscillations with respect to longitudinal lattice oscillations. Even though this mechanism, which is associated with harmonic generation due to self-interaction of the carrier waves, is today known to be of relevance in phenomena as diverse as the modulational instability and energy localization in solids, charge and information transport in biomolecules and DNA strands, and coherent signal transmission in electric lines and in optical pulse propagation 13, 14, 15, 16, 17, 18], it has only recently been considered in dusty plasmas [19] with regard to the propagation of dust-acoustic and dust-ion acoustic waves 1, 2] in a weakly-coupled (gas-like) DP. As far as the nonlinear modulation of dust-lattice oscillations is concerned, our knowledge is still at a very early stage, let alone some first attempts to model the modulation of longitudinal 20] and transverse 21] DLWs. In addition to a theoretical investigation of the occurrence of modulational instability, these studies have provided a first prediction of the possible existence of localized envelope excitations in a dust-crystal. It should be stressed that, as long known and extensively studied in solid state physics, the physics of these localized modes (basically associated with the generation of harmonics, which was recently observed experimentally; see in Ref. 22]) is essentially different from that of compressive solitons, described by Korteweg-deVries (KdV) - type models, first introduced by Melands $\varnothing$ [5] and later considered in some experiments [23, 24] and in theoretical papers [24, 25, 26, 27].

In this paper, we consider the amplitude modulation of longitudinal waves propagating in DP lattices. We will derive an equation governing the evolution of the dust lattice wave envelope and will then examine its stability with

\footnotetext{
* Preprint, submitted to Physics of Plasmas.

†On leave from: U.L.B. - Université Libre de Bruxelles, Faculté des Sciences Apliquées - C.P. 165/81 Physique Générale, Avenue F. D. Roosevelt 49, B-1050 Brussels, Belgium; also at: U.L.B., Association Euratom - Etat Belge, C.P. 231 Physique Statistique et Plasmas, Boulevard du Triomphe, B-1050 Brussels, Belgium.; Electronic address: ioannis@tp4.rub.de

‡Electronic address: ps@tp4.rub.de
} 
respect to external perturbations. Explicit conditions for the formation of different types of envelope excitations will be presented, in terms of the physical parameters involved in DP crystals and, in particular, the existence of asymmetric envelope dust-lattice solitons is predicted. This study refers to the longitudinal grain motion in an 'ideal' one-dimensional DP crystal, i.e. a single, unidimensional, infinite-sized, dust-layer of identical (in size, charge and mass) dust grains situated at spatially periodic sites (at equilibrium). It should however be noted that real crystals formed in the laboratory are often two- or three-dimensional arrays [6, 28], which may host longitudinal waves such as the ones considered herein, but also transverse (shear) waves [8] as well as dissipative nonlinear structures, as observed in recent experiments [22, 24]. Two-dimensional (2D) lattices are known from Solid State Physics to possess an enriched profile of possible excitations, including e.g. oblique (with respect to the principal axes) waves, 2D breathers and vortex excitations [29]. Therefore, this work is only a first step towards the investigation of nonlinear mechanisms in DP crystals: the mechanism put forward in this paper may be generalized to higher dimensionality; nevertheless, this is left for consideration in future work.

\section{THE MODEL}

Let us consider a layer of charged dust grains (mass $M$ and charge $q$, both assumed constant for simplicity) of lattice constant $r_{0}$. The Hamiltonian of such a chain is of the form

$$
H=\sum_{n} \frac{1}{2} M\left(\frac{d \mathbf{r}_{n}}{d t}\right)^{2}+\sum_{m \neq n} U\left(r_{n m}\right),
$$

where $\mathbf{r}_{n}$ is the position vector of the $n$-th grain; $U_{n m}\left(r_{n m}\right) \equiv q \phi(x)$ is a binary interaction potential function related to the electrostatic potential $\phi(x)$ around the $m$-th grain, and $r_{n m}=\left|\mathbf{r}_{n}-\mathbf{r}_{m}\right|$ is the distance between the $n$-th and $m$-th grains. We shall limit ourselves to considering the longitudinal $(\sim \hat{x})$ motion of the $n$-th dust grain, which obeys

$$
M\left(\frac{d^{2} x_{n}}{d t^{2}}+\nu \frac{d x_{n}}{d t}\right)=-\sum_{n} \frac{\partial U_{n m}\left(r_{n m}\right)}{\partial x_{n}} \equiv q E\left(x_{n}\right)
$$

where $E(x)=-\partial \phi(x) / \partial x$ is the electric field; the usual ad hoc damping term is introduced in the left-hand-side of Eq. (11), involving the damping rate $\nu$ due to dust-neutral collisions. A one-dimensional (1D) DP layer is considered here, but the generalization to a two-dimensional (2D) grid is straightforward. At a first step, we have omitted the external force term $F_{\text {ext }}$, often introduced to account for the initial laser excitation and/or the parabolic confinement which ensures horizontal lattice equilibrium in experiments [24].

\section{A. Equation of motion}

Assuming small displacements from equilibrium, one may Taylor expand the interaction potential $\phi(r)$ around the equilibrium inter-grain distance $l r_{0}=|n-m| r_{0}$ (between $l$-th order neighbors, $l=1,2, \ldots$ ), viz.

$$
\phi\left(r_{n m}\right)=\left.\sum_{l^{\prime}=0}^{\infty} \frac{1}{l^{\prime} !} \frac{d^{l^{\prime}} \phi(r)}{d r^{l^{\prime}}}\right|_{r=|n-m| r_{0}}\left(x_{n}-x_{m}\right)^{l^{\prime}},
$$

where $l^{\prime}$ denotes the degree of nonlinearity involved in its contribution: $l^{\prime}=1$ is the linear interaction term, $l^{\prime}=2$ stands for the quadratic potential nonlinearity, and so forth. Obviously, $\delta x_{n}=x_{n}-x_{n}^{(0)}$ denotes the displacement of the $n$-th grain from equilibrium, which now follows

$$
\begin{aligned}
M\left[\frac{d^{2}\left(\delta x_{n}\right)}{d t^{2}}+\nu \frac{d\left(\delta x_{n}\right)}{d t}\right]= & q\left\{\phi^{\prime \prime}\left(r_{0}\right)\left(\delta x_{n+1}+\delta x_{n-1}-2 \delta x_{n}\right)\right. \\
& +\left.\sum_{l^{\prime}=2}^{\infty} \frac{1}{l^{\prime} !} \frac{d \phi^{l^{\prime}+1}(r)}{d r^{l^{\prime}+1}}\right|_{r=r_{0}}\left[\left(\delta x_{n+1}-\delta x_{n}\right)^{l^{\prime}}-\left(\delta x_{n}-\delta x_{n-1}\right)^{l^{\prime}}\right] \\
& +\sum_{l=2}^{N} \phi^{\prime \prime}\left(l r_{0}\right)\left(\delta x_{n+l}+\delta x_{n-l}-2 \delta x_{n}\right)
\end{aligned}
$$




$$
\left.+\left.\sum_{l=2}^{N} \sum_{l^{\prime}=2}^{\infty} \frac{1}{l^{\prime} !} \frac{d \phi^{l^{\prime}+1}(r)}{d r^{l^{\prime}+1}}\right|_{r=l r_{0}}\left[\left(\delta x_{n+l}-\delta x_{n}\right)^{l^{\prime}}-\left(\delta x_{n}-\delta x_{n-l}\right)^{l^{\prime}}\right]\right\}
$$

We have distinguished the linear/nonlinear contributions of the first neighbors (1st/2nd lines) from the corresponding longer neighbor terms (3rd/4th lines, respectively).

Adopting the standard continuum approximation, often employed in solid state physics []ㅡ, we may assume that only small displacement variations occur between neighboring sites, i.e.

$$
\delta x_{n \pm l}=\delta x_{n} \pm l r_{0} \frac{\partial u}{\partial x}+\frac{1}{2}\left(\operatorname{lr}_{0}\right)^{2} \frac{\partial^{2} u}{\partial x^{2}} \pm \frac{1}{3 !}\left(\operatorname{lr}_{0}\right)^{3} \frac{\partial^{3} u}{\partial x^{3}}+\frac{1}{4 !}\left(\operatorname{lr}_{0}\right)^{4} \frac{\partial^{4} u}{\partial x^{4}} \pm \ldots
$$

where the displacement $\delta x(t)$ is now expressed by a continuous function $u=u(x, t)$. One may now proceed by inserting this ansatz in the discrete equation of motion (2), and carefully evaluating the contribution of each term. The calculation, quite tedious yet perfectly straightforward (to be reported, in full detail, elsewhere [30]), leads to a continuum equation of motion in the form

$$
\ddot{u}+\nu \dot{u}-v_{0}^{2} u_{x x}=v_{1}^{2} r_{0}^{2} u_{x x x x}-p_{0} u_{x} u_{x x}+q_{0}\left(u_{x}\right)^{2} u_{x x},
$$

where the subscript $x$ denotes the partial differentiation, viz. $u_{x} u_{x x}=\left(u_{x}^{2}\right)_{x} / 2$ and $\left(u_{x}\right)^{2} u_{x x}=\left(u_{x}^{3}\right)_{x} / 3$.

\section{B. Characteristic physical quantities} is

The definition of the 'sound speed', $v_{0}=\omega_{0, L} r_{0}$, associated with the longitudinal oscillation eigenfrequency $\omega_{0, L}$,

$$
v_{0}=\frac{q}{M} r_{0}^{2} \sum_{l=1}^{N} \phi^{\prime \prime}\left(l r_{0}\right) l^{2} \equiv \omega_{0, L}^{2} r_{0}^{2}
$$

where $N$ is the degree of interacting site vicinity assumed: $N=1$ for first-neighbor interactions (FNI), $N=2$ for second-neighbor interactions (SNI), and so forth. The dispersion coefficient, involving the characteristic velocity $v_{1}$, is given by

$$
v_{1}^{2} r_{0}^{2}=\frac{1}{12} \frac{q}{M} r_{0}^{4} \sum_{l=1}^{N} \phi^{\prime \prime}\left(l r_{0}\right) l^{4}
$$

We note that $v_{1}^{2}=v_{0}^{2} / 12$ for $N=1$ (only). The quadratic force (or cubic potential) nonlinearity coefficient is

$$
p_{0}=-\frac{q}{M} r_{0}^{3} \sum_{l=1}^{N} \phi^{\prime \prime \prime}\left(l r_{0}\right) l^{3}
$$

Finally, the cubic force (or quartic potential) nonlinearity coefficient $q_{0}$ is

$$
q_{0}=\frac{1}{2} \frac{q}{M} r_{0}^{4} \sum_{l=1}^{N} \phi^{\prime \prime \prime \prime}\left(l r_{0}\right) l^{4} .
$$

Let us point out that the above definitions of the coefficients in (3) are inspired by the Debye-Hückel (Yukawa) potential of the form $\phi_{D}(r)=(q / r) e^{-r / \lambda_{D}}$ (whose odd/even derivatives are negative/positive), in which case they are defined in such a way that all of $v_{0}^{2}, v_{1}^{2}, p_{0}$ and $q_{0}$ take positive values. In this case (and for $N=1$ i.e. FNI), relations (4), (5), (6) and (7) yield

$$
\begin{gathered}
\omega_{L, 0}^{2}=\frac{2 q^{2}}{M \lambda_{D}^{3}} e^{-\kappa} \frac{1+\kappa+\kappa^{2} / 2}{\kappa^{3}}=\frac{v_{0}^{2}}{\kappa^{2} \lambda_{D}^{2}}=12 \frac{v_{1}^{2}}{\kappa^{2} \lambda_{D}^{2}} \\
p_{0}=\frac{6 q^{2}}{M \lambda_{D}} e^{-\kappa}\left(\frac{1}{\kappa}+1+\frac{\kappa}{2}+\frac{\kappa^{2}}{6}\right)
\end{gathered}
$$




$$
q_{0}=\frac{12 q^{2}}{M \lambda_{D}} e^{-\kappa}\left(\frac{1}{\kappa}+1+\frac{\kappa}{2}+\frac{\kappa^{2}}{6}+\frac{\kappa^{3}}{24}\right)
$$

where we have introduced the lattice parameter $\kappa=r_{0} / \lambda_{D}$; in fact, $\kappa$ is roughly between 1 and 1.5 in DP chains spontaneously formed in current dusty plasma discharges 4, 23, 24]. These relations, which are depicted in Fig. 1] coincide with the ones in previous studies for FNI [5, 20]. Specifically, compared to the notation of Melands $\varnothing$ [see (15), (16), (26) in Ref. [5]], $v_{0}^{2}, v_{1}^{2}$ and $p_{0}$ above correspond to $v_{0}^{2}, v_{0}^{2} / 12$ and $\gamma(a) a^{3} / M$ therein. Also, compared to the notation of Amin et al. [see (1)-(4) in Ref. [20]], $v_{0}^{2}, v_{1}^{2} r_{0}^{2}$ and $p_{0}$ above correspond to $\alpha_{0}, \beta_{0}$ and $2 \gamma_{0}$ therein. However, the coefficient $q_{0}$, which can easily be seen to be practically $\approx 2 p_{0}$ or higher (see Fig. 1) - i.e. too important to be omitted - is neglected in those studies.

One should retain, for later reference, the form of the discrete dispersion relation, obtained from the general (discrete) equation of motion (2)

$$
\omega(\omega+i \nu)=\frac{4 q}{M} \sum_{l=1}^{N} \phi^{\prime \prime}\left(l r_{0}\right) \sin ^{2}\left(\frac{l k r_{0}}{2}\right)=\frac{4 q}{M} \sum_{l=1}^{N} \phi^{\prime \prime}\left(l \kappa \lambda_{D}\right) \sin ^{2}\left[\frac{l \kappa\left(k \lambda_{D}\right)}{2}\right] .
$$

One may readily verify that the standard $1 \mathrm{D}$ acoustic wave dispersion relation $\omega \approx k v_{0}$ is obtained in the small $k$ (long wavelength) limit: check by setting $\sin \left(l k r_{0} / 2\right) \approx l k r_{0} / 2$ (and recalling the general definition of $v_{0}$ above). For FNI and Debye-Hückel interactions, one obtains

$$
\omega(\omega+i \nu)=\frac{4 q}{M} \phi^{\prime \prime}\left(r_{0}\right) \sin ^{2} \frac{k r_{0}}{2} \equiv 4 \omega_{L, 0}^{2} \sin ^{2} \frac{k r_{0}}{2}
$$

[cf. (4) for $N=1$ ]. We note that the LDLW frequency and the sound speed will be of the order of: $\hat{\omega}_{0}=$ $\left[2 q^{2} /\left(M \lambda_{D}^{3}\right)\right]^{1 / 2}\left(\equiv \omega_{0} \sqrt{2}=\omega_{\max }\right)$ and $\hat{v}_{0}=\left[2 q^{2} /\left(M \lambda_{D}\right)\right]^{1 / 2} \equiv \omega_{0} \lambda_{D}^{2}$, respectively; these characteristic quantities scale as $\hat{\omega}_{0} \sim r^{-1 / 2} a^{-3 / 2} U$ and $\hat{\omega}_{0} \sim r^{-1 / 2} a^{-1 / 2} U$, respectively, for a given dust grain radius $r$, an inter-grain separation $a$ and the dust surface potential $U=q / r$ (following Ref. [5]).

Even though all the formulae above are provided in their general form, in the following we shall assume that $N=1$ (FNI) and will also set $\nu \approx 0$ at a first step, for the sake of analytical tractability. These assumptions will be relaxed in a forthcoming work, also accounting for higher-order nonlinearities neglected here, which may be evaluated in a systematic manner [30]. Our aim here is to emphasize on a physical mechanism, rather than putting forward a tedious and exhaustive mathematical model.

\section{MULTIPLE SCALE EXPANSION - DERIVATION OF A NONLINEAR SCHRÖDINGER EQUATION}

According to the standard reductive perturbation method [11, 31], we shall consider a small displacement of the form: $u \rightarrow 0+\epsilon u_{1}+\epsilon^{2} u_{2}+\ldots$, where $\epsilon \ll 1$ is a small parameter, and solutions $u_{n}$ at each order are assumed to be a sum of $m$-th order harmonics, viz. $u_{n}=\sum_{m=0}^{n} u_{m}^{(n)} \exp [i(k x-\omega t)]$ (the reality condition $u_{-m}^{(n)}=u_{m}^{(n)^{*}}$ is understood). Time and space scales are accordingly expanded as

$$
\partial / \partial t \rightarrow \partial / \partial T_{0}+\epsilon \partial / \partial T_{1}+\epsilon^{2} \partial / \partial T_{2}+\ldots \equiv \partial_{0}+\epsilon \partial_{1}+\epsilon^{2} \partial_{2}+\ldots,
$$

and

$$
\partial / \partial x \rightarrow \partial / \partial X_{0}+\epsilon \partial / \partial X_{1}+\epsilon^{2} \partial / \partial X_{2}+\ldots \equiv \nabla_{0}+\epsilon \nabla_{1}+\epsilon^{2} \nabla_{2}+\ldots
$$

implying that

$$
\begin{gathered}
\partial^{2} / \partial t^{2} \rightarrow \partial_{0}^{2}+2 \epsilon \partial_{0} \partial_{1}+\epsilon^{2}\left(\partial_{1}^{2}+2 \partial_{0} \partial_{2}\right)+\ldots \\
\partial^{2} / \partial x^{2} \rightarrow \nabla_{0}^{2}+2 \epsilon \nabla_{0} \nabla_{1}+\epsilon^{2}\left(\nabla_{1}^{2}+2 \nabla_{0} \nabla_{2}\right)+\ldots
\end{gathered}
$$

and

$$
\partial^{4} / \partial x^{4} \rightarrow \nabla_{0}^{4}+4 \epsilon \nabla_{0}^{3} \nabla_{1}+2 \epsilon^{2} \nabla_{0}^{2}\left(3 \nabla_{1}^{2}+2 \nabla_{0} \nabla_{2}\right)+\ldots
$$

We now proceed by substituting all the above series in (3) and isolating terms arising in the equation of motion at each order in $\epsilon^{n}$. By solving for $u_{n}$, then substituting in the following order $\epsilon^{n+1}$ and so forth, we obtain the $m$-th harmonic amplitudes $u_{n}^{(m)}$ in each order, along with a compatibility condition up to any given order. 
The equation obtained in order $\sim \epsilon^{1}$ is

$$
\left(\partial_{0}^{2}-v_{0}^{2} \nabla_{0}^{2}-v_{1}^{2} r_{0}^{2} \nabla_{0}^{4}\right) u_{1} \equiv L_{0} u_{1}=0 .
$$

We may assume that $u_{1}=u_{1}^{(1)} \exp [i(k x-\omega t)]+c . c . \equiv \exp (i \theta)+c . c$. , where $\omega, k=2 \pi / \lambda$ and $\lambda$ denote the carrier wave frequency, wavenumber and wavelength, respectively; c.c. stands for the complex conjugate of the preceding quantity everywhere.

The dispersion relation obtained from (13) is of the form

$$
\omega^{2}=k^{2} v_{0}^{2}-k^{4} v_{1}^{2} r_{0}^{2}=k^{2} v_{0}^{2}\left(1-\frac{v_{1}^{2}}{v_{0}^{2}} k^{2} r_{0}^{2}\right),
$$

predicting an acoustic behaviour (viz. $\omega \approx k v_{0}$ ) for very low $k$, and dispersion (weighted by $v_{1}^{2} r_{0}^{2}$ ) at higher values. Notice that the reality condition $\omega>0$, implying $k<\left(v_{0} / v_{1}\right) r_{0}^{-1} \equiv k_{c r, 1}\left(=\sqrt{12} r_{0}^{-1}\right.$ for FNI), is in principle satisfied, covered by the continuum hypothesis $k \ll\left(\pi / r_{0}\right) \equiv k_{c r, 0}$. Specifically, one may numerically verify the agreement between the discrete dispersion relation in (12) (for $\nu=0$ ) and its continuum approximation (14) - see Fig. 2 for instance, aiming at an accuracy interval of $99 \%$ (90\%), i.e. allowing for a relative error of $1 \%(10 \%)$, one should restrict the confidence interval of this study to values of $k r_{0}$ below 0.3 (1.0).

Let us evaluate the action of the linear operator $L_{0}$, defined above, on higher harmonics of the phase $\theta$

$$
L_{0} e^{i n \theta}=\left[(-i n \omega)^{2}-v_{0}^{2}(i n k)^{2}-v_{1}^{2} r_{0}^{2}(i n k)^{4}\right] e^{i n \theta}=\ldots=-n^{2}\left(n^{2}-1\right) v_{1}^{2} r_{0}^{2} k^{4} e^{i n \theta} \equiv D_{n} e^{i n \theta},
$$

where we made use of the dispersion relation (14). We observe that $D_{0}=D_{1}=0$.

To order $\sim \epsilon^{2}$, we have

$$
L_{0} u_{2}=-2\left(\partial_{0} \partial_{1}-v_{0}^{2} \nabla_{0} \nabla_{1}-2 v_{1}^{2} r_{0}^{2} \nabla_{0}^{3} \nabla_{1}\right) u_{1}-p_{0}\left(\nabla_{0} u_{1}\right)\left(\nabla_{0}^{2} u_{1}\right) .
$$

One now has to impose the condition of suppression of secular terms (i.e. terms $\sim e^{i \theta}$ in the right-hand side, which may resonate with the null space of the operator $L_{0}$ ), which takes the form

$$
\frac{\partial u_{1}^{(1)}}{\partial T_{1}}+v_{g} \frac{\partial u_{1}^{(1)}}{\partial X_{1}}=0
$$

or $\partial u_{1}^{(1)} / \partial \zeta=0$, i.e. $u_{1}^{(1)}=u_{1}^{(1)}(\xi, \tau)$, where $\zeta=\epsilon\left(x-v_{g} t\right)$ and $\tau=\epsilon^{2} t$, implying that the slowly-varying wavefront (envelope) travels at the group velocity $v_{g}=\omega^{\prime}(k)=\left(v_{0}^{2}-2 v_{1}^{2} r_{0}^{2} k^{2}\right) k / \omega$. We notice that $v_{g}$ becomes zero at $k=v_{0} /\left(v_{1} \sqrt{2}\right) r_{0}^{-1} \equiv k_{c r, 1} / \sqrt{2}\left(=\sqrt{6} r_{0}^{-1}\right.$ for FNI), beyond which it becomes negative: the wave envelope and the carrier wave then propagate in opposite directions (backward wave). Nevertheless, for the sake of rigor, note that this comment rather becomes obsolete once one properly takes into account the influence of the lattice discreteness on the form of the dispersion relation $\omega=\omega(k)$; see Fig. [2

The solution $u_{2}$ obtained at this order involves a second harmonic contribution $u_{2}^{(2)} \exp (2 i \theta)=$ $\left(i p_{0} k^{3} / D_{2}\right)\left(u_{1}^{(1)}\right)^{2} \exp (2 i \theta)=-i\left[p_{0} /\left(12 v_{1}^{2} r_{0}^{2} k\right)\right]\left(u_{1}^{(1)}\right)^{2} \exp (2 i \theta)$, as well as a zeroth (and possibly a first) harmonic(s), which may be determined by higher-order relations.

One may now proceed to the next (third) order in $\epsilon$. The condition for annihilation of secular terms now takes the form

$$
i \frac{\partial u_{1}^{(1)}}{\partial \tau}+P \frac{\partial^{2} u_{1}^{(1)}}{\partial \zeta^{2}}+Q_{0}\left|u_{1}^{(1)}\right|^{2} u_{1}^{(1)}+\frac{p_{0} k^{2}}{2 \omega} u_{1}^{(1)} \frac{\partial u_{0}^{(1)}}{\partial \zeta}=0 .
$$

The dispersion coefficient $P$ is

$$
P=\frac{1}{2} \omega^{\prime \prime}(k)=-\frac{1}{2 \omega}\left(v_{g}^{2}-v_{0}^{2}+6 v_{1}^{2} r_{0}^{2} k^{2}\right)=\frac{v_{1}^{4} r_{0}^{4} k^{4}}{\omega^{3}}\left(k^{2}-\frac{3 v_{0}^{2}}{2 v_{1}^{2} r_{0}^{2}}\right)
$$

while the nonlinearity coefficient $Q_{0}$ is

$$
Q_{0}=-\frac{k^{2}}{2 \omega}\left(q_{0} k^{2}+\frac{p_{0}^{2}}{6 v_{1}^{2} r_{0}^{2}}\right)
$$


For $q_{0}=0$ and $u_{0}^{(1)}=0$, these expressions agree exactly with Eqs. (17) and (18) in Ref. [20]. However, the zerothharmonic correction $u_{0}^{(1)}$ cannot be set equal to zero; indeed, collecting the constant (zeroth-harmonic) terms arising in this order, one obtains

$$
\frac{\partial^{2} u_{0}^{(1)}}{\partial \zeta^{2}}=-\frac{p_{0} k^{2}}{v_{g}^{2}-v_{0}^{2}} \frac{\partial}{\partial \zeta}\left|u_{1}^{(1)}\right|^{2},
$$

which may immediately be integrated, yielding

$$
\frac{\partial u_{0}^{(1)}}{\partial \zeta}=-\frac{p_{0} k^{2}}{v_{g}^{2}-v_{0}^{2}}\left|u_{1}^{(1)}\right|^{2}+C \equiv R(k)\left|u_{1}^{(1)}\right|^{2}+C,
$$

where $C$ is a constant, to be determined by the boundary conditions (e.g. $C=0$ if $u_{1}^{(1)}=0$ at $x= \pm \infty$ ). Note that the resonance condition $v_{g}=v_{0}$ amounts to $k^{2}-3 v_{0}^{2} /\left(4 v_{1}^{2} r_{0}^{2}\right)=0$, which is not satisfied in the parameter range of the continuum approximation; on the contrary, $v_{g}<v_{0}$ everywhere, implying that $R(k)$ takes positive/negative values for positive/negative $p_{0}$ : for Debye interactions, $p_{0}>0$ and $R>0$. The non-vanishing constant term $u_{0}^{(1)}$ is purely due to cubic interaction potential nonlinearity (and disappears for $p_{0}=0$ ). This is known in solid state physics 11,12 .

Eqs. (18) and (22) form a coupled set of evolution equations, governing the dynamics at first order in $\epsilon$. They may readily be combined into the form of a Nonlinear Schrödinger Equation (NLSE)

$$
i \frac{\partial A}{\partial \tau}+P \frac{\partial^{2} A}{\partial \zeta^{2}}+Q|A|^{2} A=0,
$$

which, once solved for the amplitude of the first harmonic $A=u_{1}^{(1)}$, immediately provides the solution for $u_{0}^{(1)}$ upon substitution into (22). A linear term $\sim C A$ has been neglected, since it is canceled by a trivial phase shift transformation. The final form of the nonlinearity coefficient $Q$ now reads

$$
Q=-\frac{k^{2}}{2 \omega}\left(q_{0} k^{2}+\frac{p_{0}^{2}}{6 v_{1}^{2} r_{0}^{2}}\right)-\frac{p_{0}^{2} k^{4}}{2 \omega} \frac{1}{v_{g}^{2}-v_{0}^{2}} .
$$

In the infinite wavelength limit (i.e. near $k \approx 0$ ), we have

$$
P \approx-\frac{3 v_{1}^{2} r_{0}^{2}}{2 v_{0}} k-\frac{5 v_{1}^{4} r_{0}^{4}}{4 v_{0}^{3}} k^{3}+\mathcal{O}\left(k^{5}\right)
$$

and

$$
Q \approx \frac{p_{0}^{2}}{12 v_{1}^{2} r_{0}^{2} v_{0}} k+\left(\frac{7 p_{0}^{2}}{72 v_{0}^{3}}-\frac{q_{0}}{2 v_{0}}\right) k^{3}+\mathcal{O}\left(k^{5}\right) .
$$

Let us stress that the extra term, which makes the difference between $Q_{0}$ and $Q$, changes completely the behaviour of the nonlinearity coefficient near $k \approx 0$. For example, we find that

$$
Q_{0} \approx-\frac{p_{0}^{2}}{12 v_{1}^{2} r_{0}^{2} v_{0}} k-\left(\frac{p_{0}^{2}}{24 v_{0}^{3}}+\frac{q_{0}}{2 v_{0}}\right) k^{3}+\mathcal{O}\left(k^{5}\right) .
$$

Note, once more, that the results in this Section are general and apply to a wide class of physical problems. In any given problem, involving an arbitrary (analytical, possibly long-range) interaction law, Eqs. (18), (22) and (23) provide the evolution of the first-order correction $u_{1}$ to the particle displacement $u$, if the latter is governed by an equation of motion of the form of (2), as expressed in the continuum limit by Eq. (3). To first order in $\epsilon$, the solution then reads

$$
u(x, t) \approx \epsilon\left[u_{0}^{(1)}(\zeta, \tau)+u_{1}^{(1)}(\zeta, \tau)+c . c .\right]+\mathcal{O}\left(\epsilon^{2}\right),
$$

where $u_{0}^{(1)}$ is given by (22) (it vanishes if, and only if, $p_{0}=0$ ) and $u_{1}^{(1)}$ corresponds to (the amplitude of) a small translation and an oscillation, respectively, both traveling at the group velocity $v_{g}$. We emphasize, as a general result, that the existence of a non-zero cubic potential nonlinearity term $p_{0}$ implies the existence of a finite translation (zeroth-harmonic) correction $u_{0}^{(1)}$ to first order in $\epsilon$, while the sign of $p_{0}$ affects the form of $u_{0}^{(1)}$ (i.e. the lattice compression or rarefaction); furthermore, it also determines the (sign of the) second-harmonic correction, as we saw above. Nevertheless, the sign of $p_{0}$ does not affect the form of the coefficients in (23), and thus neither the plane wave stability profile. 


\section{STABILITY ANALYSIS}

The standard stability analysis [18, 32, 33] consists in linearizing around the (Stokes) plane wave solution of (23), $A=\hat{A} e^{i Q|\hat{A}|^{2} \tau}+$ c.c. (notice the amplitude dependence of the frequency), by setting $\hat{A}=\hat{A}_{0}+\epsilon \hat{A}_{1}$ and taking the perturbation $\hat{A}_{1}$ to be of the form $\hat{A}_{1}=\hat{A}_{1,0} e^{i(\hat{k} \zeta-\hat{\omega} \tau)}+$ c.c., where $\hat{k}$ and $\hat{\omega}$ denote the perturbation's wavenumber and frequency, to be distinguished from the carrier wave's homologous quantities, $k$ and $\omega$. Hence from (23) one readily obtains the dispersion relation $\hat{\omega}^{2}=P^{2} \hat{k}^{2}\left(\hat{k}^{2}-2 \frac{Q}{P}\left|\hat{A}_{0}\right|^{2}\right)$. The wave will thus be stable if the product $P Q$ is negative. However, for positive $P Q>0$, instability sets in for wavenumbers below a critical value $\hat{k}_{c r}=\sqrt{2 \frac{Q}{P}}\left|\hat{A}_{0}\right|$, i.e. for wavelengths above a threshold: $\lambda_{c r}=2 \pi / \hat{k}_{c r}$, with an increment $\sigma=|\operatorname{Im} \hat{\omega}(\hat{k})|$ which attains a maximum value at $\hat{k}=\hat{k}_{c r} / \sqrt{2}$, viz.

$$
\sigma_{\max }=|\operatorname{Im} \hat{\omega}|_{\hat{k}=\hat{k}_{c r} / \sqrt{2}}=|Q|\left|\hat{A}_{0}\right|^{2} .
$$

It turns out that the instability condition depends only on the sign of the product $P Q$, which may be studied numerically by using the expressions derived above.

Let us go back to the expressions (19) and (24) for the coefficients $P$ and $Q$. First, we notice that $P$ is always negative, given the condition $k<k_{c r, 1}=\left(v_{0} / v_{1}\right) r_{0}^{-1}$ imposed above. On the other hand, the nonlinearity coefficient $Q$ may take either negative or positive values, depending on the physical parameters $v_{0}, v_{1}, p_{0}$ and $q_{0}$ and, of course, the wavenumber $k$. To see this, one may cast (24) in the form

$$
Q=-\frac{k^{2}}{2 \omega}\left[q_{0} k^{2}-\frac{3-2 v_{1}^{2} r_{0}^{2} k^{2} / v_{0}^{2}}{3-4 v_{1}^{2} r_{0}^{2} k^{2} / v_{0}^{2}} \frac{p_{0}^{2}}{6 v_{1}^{2} r_{0}^{2}}\right],
$$

and then examine its sign in terms of $k$. This can be done either by a straightforward (yet rather lengthy) analytical calculation (omitted here) or numerically (see below). Notice, as a general remark, the pole created at the resonance point $v_{g}=v_{0}$, for $k=v_{0} \sqrt{3} /\left(2 v_{1}\right) r_{0}^{-1}\left(=3 r_{0}^{-1}\right.$ for $\left.\mathrm{FNI}\right)$. In conclusion, the carrier wave will be modulationally unstable for $k$ values corresponding to $Q<0(P Q>0)$, and stable for $Q>0(P Q<0)$. These results are valid for any given form of the electrostatic interaction potential $\phi(r)$ and neighboring site vicinity $N$, which (both) in fact enter the physical parameters via the expressions (4) - (7).

Let us now focus on the dust-lattice problem. At a first step, one may explicitly assume first neighbor DebyeHückel type interactions, by substituting with expressions (8) - (10) into the above relations for $P, Q$, and then study the behaviour of all the relevant physical quantities as a function of, say, the lattice parameter $\kappa$. Notice that the stability profile deduced above is dramatically modified, in comparison with the limit of vanishing cubic interaction nonlinearity, i.e. $q_{0}=0$, considered in Ref. [20], where the instability of plane waves was directly prescribed (in addition to $u_{0}^{(1)}=0$ ). A simple numerical investigation shows that $Q$ is always positive (prescribing stability, since $P<0$ ) at low values of the wavenumber $k$; see Figures 3 4 This is true everywhere, for $k$ below the resonance threshold $k r_{0} \approx 3$, except for $\kappa$ of the order of $\approx 0.75 r_{0}^{-1}$ or lower, where a small instability region is encountered; see Fig. 4 [notice the asymptotic curve at $k r_{0}=v_{0} \sqrt{3} /\left(2 v_{1}\right)=3$, as expected from (25), beyond which the continuum approximation certainly fails]. Long wavelength LDLWs are therefore expected to be stable, while shorter wavelength LDLWs may be unstable. For rigor, it should be admitted that the instability (carrier wavenumber) threshold $k_{c r}$ is quite high, and rather beyond the validity of this continuum model (recall the assumption made above, that $k \ll k_{c r, 0}=\pi / r 0$ ). Therefore, while small $k$ stability may be taken for granted, we only possess an indication for (i.e. not a rigorous proof of) instability for longer $k$. We draw the conclusion that LDL waves are most likely to remain stable with respect to external perturbations (even though this statement may a priori not be valid for a different interaction potential). The present results are in agreement with previous prediction in atomic chains [11, 12].

\section{NONLINEAR EXCITATIONS}

Equation (23) is an integrable nonlinear partial differential equation [33, 34], which is known to possess different types of localized, constant profile, travelling wave solutions (solitons). Several types of such localized modes are presented in Refs. [12, 32, 34, 35]) (see Ref. [35] for a review) so we will only briefly outline the analytical form of those we are interested in, and discuss their relevance to our problem.

A solution of Eq. (23) may be sought in the form $A(\zeta, \tau)=\rho(\zeta, \tau) e^{i \Theta(\zeta, \tau)}$, where $\rho$ and $\sigma$ are real variables to be determined. Different types of solution are thus obtained, depending on the sign of the product $P Q$. Once $u_{1}^{(1)}=A$ is thus determined, the zeroth-harmonic first-order correction $u_{0}^{(1)}$ is obtained from (22). 
For $P Q>0$ we find the bright envelope soliton 36 ]

$$
A(x, t)=\rho_{0} \operatorname{sech}\left[\left(x-v_{e} t\right) / L_{e}+x_{e}\right] \exp \left[-2 i \alpha\left(x-v_{g} t\right)-4\left(\alpha^{2}-\eta^{2}\right) P t\right]
$$

$(\operatorname{sech} x=1 / \cosh x)$, which represents a localized envelope pulse travelling at a speed $v_{e}=v_{g}-4 \alpha P$. The pulse width $L_{e}=1 /(2 \eta)$ is related to the (constant) maximum amplitude $\rho_{0}=2 \eta(P / Q)^{1 / 2}$ as $\rho_{0}=(P / Q)^{1 / 2} / L_{e}$. See that both of the small (arbitrary) parameters $\alpha$ and $\eta$ bear dimensions of inverse length. Substituting (26) into (22), we obtain the zeroth-harmonic term $u_{0}^{(1)}=R \int\left|u_{1}^{(1)}\right|^{2} d \zeta=2 \eta P R(k) / Q \tanh \left[\left(x-v_{e} t\right) / L_{e}+x_{e}\right]+c$ (we will assume that $c=0)$. Combining the preceding formulae, we obtain the asymmetric bright-envelope modulated solution for the (total) dust-grain displacement (to order $\sim \epsilon$ )

$$
u_{1}=A_{0} \operatorname{sech}\left[\left(x-v_{e} t\right) / L_{e}+c_{e}\right] \cos \left[\left(x-v_{c} t\right) / L_{0}+c_{0}\right]+B_{0} \tanh \left[\left(x-v_{e} t\right) / L_{e}+c_{e}\right]
$$

where $A_{0}=2 \rho_{0}=4 \eta(P / Q)^{1 / 2}, B_{0}=R(k) \rho_{0}^{2} L_{e}=2 \eta R(k) P / Q$ and the shifted (modulated) carrier wavenumber $k_{c}=L_{0}^{-1}$ and frequency $\omega_{c}=v_{0} / L_{0}$ now depend on: $L_{0}=1 /(k-2 \alpha)$ and $v_{c}=\left[\omega-2 \alpha v_{g}+4 P\left(\alpha^{2}-\eta^{2}\right)\right] L_{0}$; $c_{e}$ and $c_{e}$ are arbitrary real constants. Equation (27) represents a superposition of a localized modulated harmonic oscillation and a kink-like excitation, which is characterized by different constant asymptotic limits (at $x= \pm \infty$ ). Since $R(k)>0$ in a Debye crystal, this solution will therefore represent a localized compression co-propagating with a strongly modulated envelope oscillation; see Fig. [5.

For $P Q<0$, we have the grey envelope soliton [36]

$$
A(\zeta, \tau)=\rho_{1}\left\{1-a^{2} \operatorname{sech}^{2}\left[(\zeta-2 \alpha \tau) / L_{1}\right]\right\}^{1 / 2} \exp [i \sigma(\zeta, \tau)]
$$

where

$$
\sigma(\zeta, \tau)=\sin ^{-1}\left\{a \tanh \left(\zeta / L_{1}\right)\left[1-a^{2} \operatorname{sech}^{2}\left(\zeta / L_{1}\right)\right]^{-1 / 2}-2 \alpha \zeta+\left(\Omega-4 P \alpha^{2}\right) \tau\right\}
$$

which represents a localized region of negative wave density (a void), with finite amplitude $(1-a) \rho_{1}$ at $\zeta=0$; $0 \leq a \leq 1$ ) [one may choose $\Omega=4 P \eta^{2}$ and $\rho_{1}=2 \eta(|P / Q|)^{1 / 2}$, for analogy with (26)]. Again, the pulse width $L_{1}=(|P / Q|)^{1 / 2} /\left(a \rho_{1}\right)$ is inversely proportional to the amplitude $\rho_{1}$. Going back to the original variables, we obtain the dust grain harmonic oscillation

$$
u_{1}^{(1)} \exp i \theta+c . c .=A_{1}\left\{1-a^{2} \operatorname{sech}^{2}\left[\left(x-v_{e} t\right) / L_{1}+c_{e}\right]\right\}^{1 / 2} \cos \left[\left(x-v_{c} t\right) / L_{0}+\sigma_{1}(x, t)\right],
$$

which has a maximum amplitude $A_{1}=2 \rho_{1}$ and a phase shift

$$
\sigma_{1}(x, t)=\sin ^{-1}\left[a \tanh \left(x-v_{e} t\right) / L_{1}\right]\left[1-a^{2} \operatorname{sech}^{2}\left(x-v_{e} t\right) / L_{1}\right]^{-1 / 2}+\sigma_{0},
$$

where $v_{e}, v_{c}$ and $L_{0}$ are just as defined previously. Notice the (dimensionless) parameter $a$, which regulates the depth of the excitation. For $a=1$, one obtains the dark envelope soliton

$$
u_{1}^{(1)} \exp i \theta+c . c .=A_{1} \tanh \left[\left(x-v_{e} t\right) / L_{1}+c_{e}\right] \cos \left[\left(x-v_{c} t\right) / L_{0}+c_{0}\right],
$$

which describes a localized density hole, characterized by a vanishing amplitude at $\zeta=0$; see Fig. 6 The darkor grey-soliton-modulated wavepackets in the previous paragraph, are superposed on a constant (zeroth-harmonic) displacement, given by (22) as

$$
u_{0}^{(1)}=-B_{1} \tanh \left[\left(x-v_{e} t\right) / L_{1}+c_{e}\right]
$$

where $B_{1}=R(k) \rho_{1}^{2} a^{2} L_{1}=a R(k)(|P / Q|)^{1 / 2} \rho_{1}$; the constant $C$ in (22) was chosen as $C=-R(k) \rho_{1}^{2}$ for constant asymptotic values at infinity. Combining the latter formulae, we obtain the asymmetric dark/grey modulated envelope solution for the dust-grain total displacement (to order $\sim \epsilon$ )

$$
u_{1}=A_{1}\left\{1-a^{2} \operatorname{sech}^{2}\left[(\zeta-2 \alpha \tau) / L_{1}\right]\right\}^{1 / 2} \cos \left[\left(x-v_{c} t\right) / L_{0}+\sigma+c_{0}\right]-B_{1} \tanh \left[\left(x-v_{e} t\right) / L_{e}+c_{e}\right]
$$

or, for $a=1$,

$$
u_{1}=A_{1} \tanh \left[\left(x-v_{e} t\right) / L_{1}+c_{e}\right] \cos \left[\left(x-v_{c} t\right) / L_{0}+c_{0}\right]-B_{1} \tanh \left[\left(x-v_{e} t\right) / L_{e}+c_{e}\right]
$$

where all quantities were defined previously. For $R>0$, as in the Debye DP crystal case, the dark/grey type excitations correspond to a localized density dip accompanied by a rarefaction propagating in the lattice; see Fig. 6] 
A quantity of importance comes out to be the ratio $\xi=P / Q$ : its sign dictates the type of envelope soliton which should, in principle, propagate in the lattice, while (the square root of) its absolute value represents a measure of the soliton amplitude $A_{0}$ for a given width $L$, viz. $A_{0}=\sqrt{\xi} / L$. In figure 7 we have depicted $\xi$ versus the (normalized) wavenumber $k r_{0}$ and the lattice parameter $\kappa$. We notice an increase of the absolute value of the (negative, mostly) ratio with decreasing $\kappa$ and with increasing $k$. One draws the conclusion that for a given excitation width $L$ (assumed $L \gg r_{0}$, according to our approximation), shorter wavelength (i.e. higher wavenumber) carrier waves will form dark-type excitations (pulses) with an increased maximum displacement $A_{0}$; this picture, which is true below, say, $k \approx 2.25 r_{0}^{-1}$, is inverted above this value (nevertheless, there may be some doubt above the validity of results in that region, as discussed above). Also, higher values of $\kappa$, i.e. higher lattice constant $r_{0}$ values for a given Debye length $\lambda_{D}$, seem to favor narrower excitations (as compared to $r_{0}$ ). Notice, in Fig. $7 \mathrm{~h}$, the curly-shaped curve at the bottom, indicating the poles of the ratio $P / Q$, i.e. where $Q=0$ ( $\xi>0$ only below this curve), as well as the resonance asymptotic line at $k r_{0}=3$. See that positive values of $\xi$ are confined in a small, rather short-wavelength and small $\kappa$ region; see Fig. $17 \mathrm{~h}$. As a final comment, notice that values deduced from Fig. 7 are in agreement with the continuum approximation. For instance, as a crude estimation, taking $L=5 r_{0}$ and $\kappa \approx 1$, one obtains $\xi \approx 0.025 r_{0}^{2}$, implying $A_{0}=\sqrt{\xi} / L \approx 0.03 r_{0}$, i.e. $A_{0} \ll r_{0}$ as predicted.

Concluding, the instability/stability regions depicted e.g. in Figs. 3 4 in fact also limit the $(k, \kappa)$ parameter pair values where bright (dark/grey) solutions i.e. density pulses (holes) may exist. Furthermore, the envelope characteristics will depend on the carrier wave dispersion via $P$ and $Q$; for instance, regions with lower values of $P$ (or higher values of $Q$ ) will support narrower excitations. Admittedly, however, dust lattices will rather not favor bright-type structure formation by unstable long-wavelength LDL waves. On the other hand, such waves will mostly be stable to external perturbations, and may locally form density dips (envelope holes).

\section{CONCLUSIONS}

In this paper, we have investigated the amplitude modulation of longitudinal dust-lattice waves propagating in a one-dimensional dust-crystal. By using a continuum approximation, we have derived a Nonlinear Schrödinger Equation governing the evolution of the LDL wave envelope. We have found that long wavelength carrier waves will generally be stable, possibly evolving towards the formation of voids, i.e. asymmetric dark/grey-type coherent envelope structures, while longer wavelengths may allow for the formation of envelope pulses (asymmetric brighttype envelope excitations). Furthermore, we observe that the total grain displacement will bear a (weak) constant displacement (zeroth mode), due to the intrinsic third-order nonlinearity of the Debye potential. It may be admitted that the former (dark) excitations may rather not be relevant to this idealized, infinite chain model, since they correspond to an infinite amount of energy stored in the lattice. Nevertheless, they may be taken as a hint towards excitations sustained in finite-length, highly discrete, real lattices.

The present study aims in making a first step towards the elucidation of the nonlinear harmonic generation mechanisms dominating weakly-nonlinear longitudinal wave packets in dust-crystals. The formation of LDLW-related structures has thus been predicted, and will hopefully be confirmed by appropriately designed experiments or by numerical simulations. In fact, through the present investigation, a link has been established towards the formalism of discreteness-related intrinsic localized modes (discrete breathers) widely studied recently in solid state systems [37, 38]. This work should, in principle, be extended by including realistic effects associated with crystal asymmetries, defects, dust charging, ion-drag and multiple dust-layer coupling [39]. In particular, one should consider a similar effect in more realistic, e.g. 2D, geometries, as the ones observed in recent experiments with laser-excited dust lattices 22, 24]. Concluding, this work was devoted to a nonlinear mechanism for harmonic generation and coherent structure formation. Although here presented in a $1 \mathrm{D}$ geometry, for simplicity, this qualitative mechanism may be generalized to $2 \mathrm{D}$ lattices; this possibility will be considered in future work.

\section{Acknowledgments}

This work was partially supported by the European Commission (Brussels) through the Human Potential Research and Training Network via the project entitled "Complex Plasmas: The Science of Laboratory Colloidal Plasmas and Mesospheric Charged Aerosols" (Contract No. HPRN-CT-2000-00140). 
[1] F. Verheest, Waves in Dusty Space Plasmas (Kluwer Academic Publishers, Dordrecht, 2001).

[2] P. K. Shukla and A. A. Mamun, Introduction to Dusty Plasma Physics (Institute of Physics, Bristol, 2002).

[3] C. Kittel, Introduction to Solid State Physics (John Wiley and Sons, New York, 1996).

[4] J. Chu and L. I, Phys. Rev. Lett. 72, 4009 (1994); H. Thomas, G. E. Morfill and V. Demmel, Phys. Rev. Lett. 73, 652 (1994); A. Melzer, T. Trottenberg and A. Piel, Phys. Lett. A 191, 301 (1994); Y. Hayashi and K. Tachibara, Jpn. J. Appl. Phys. 33, L84 (1994).

[5] F. Melandsø, Phys. Plasmas 3, 3890 (1996).

[6] G. E. Morfill, H. M. Thomas and M. Zuzic, in Advances in Dusty Plasma Physics, Eds. P. K. Shukla, D. A. Mendis and T. Desai (World Scientific, Singapore, 1997), p. 99; G. E. Morfill, H. M. Thomas, U. Konopka, H. Rothermel, M. Zuzic, A. Ivlev and J. Goree, Phys. Rev. Lett. 83, 1598 (1999).

[7] B. Farokhi, P. K. Shukla, N. L. Tsintsadze and D. D. Tshkakaya, Phys. Lett. A 264, 318 (1999); idem, Phys. Plasmas 7, $814(2000)$.

[8] S. Nunomura, D. Samsonov and J. Goree, Phys. Rev. Lett. 84, 5141 (2000).

[9] D. D. Tskhakaya and P. K. Shukla, Phys. Lett. A 286, 277 (2001).

[10] X. Wang and A. Bhattacharjee, Phys. Plasmas 6 (11), 4388 (1999); X. Wang, A. Bhattacharjee and S. Hu, Phys. Rev. Lett. 86, 2569 (2001).

[11] A. Tsurui, Progr. Theor. Phys. 48, 1196 (1972).

[12] N. Flytzanis, St. Pnevmatikos and M. Remoissenet, J. Phys. C: Solid State Phys. 18, 4603 (1985).

[13] A. C. Scott and D. W. McLaughlin, Proc. IEEE 61 (10), 1443 (1973).

[14] I. Daumont, T. Dauxois and M. Peyrard, Nonlinearity10, 617 (1997); M. Peyrard, Physica D 119, 184 (1998).

[15] A. S. Davydov, Solitons in Molecular Systems (Reidel Publ. - Kluwer, Dordrecht, 1985).

[16] A. Hasegawa, Optical Solitons in Fibers (Springer-Verlag, 1989).

[17] A. C. Newell and J. V. Moloney, Nonlinear Optics (Addison-Wesley Publ. Co., Redwood City Ca., 1992).

[18] M. Remoissenet, Waves Called Solitons (Springer-Verlag, Berlin, 2nd Ed., 1996).

[19] M. R. Amin, G. E. Morfill and P. K. Shukla, Phys. Rev. E 58, 6517 (1998); I. Kourakis and P. K. Shukla, Phys. Plasmas 10 (9), 3459 (2003); I. Kourakis and P. K. Shukla, "Oblique amplitude modulation of dust-acoustic plasma waves", Physica Scripta 69, in press (2004).

[20] M. R. Amin, G. E. Morfill and P. K. Shukla, Phys. Scripta 58, 628 (1998).

[21] I. Kourakis and P. K. Shukla, "Weakly nonlinear effects associated with transverse oscillations in dusty plasma crystals", Physica Scripta, in press (2004).

[22] S. Nunomura, S. Zhdanov, G. Morfill and J. Goree, Phys. Rev. E 68, 026407 (2003).

[23] V. Nosenko, S. Nunomura and J. Goree, Phys. Rev. Lett. 88, 215002 (2002).

[24] D. Samsonov, A. V. Ivlev, R. A. Quinn, G. Morfill and S. Zhdanov, Phys. Rev. Lett. 88, 095004 (2002).

[25] S. K. Zhdanov, D. Samsonov and G. Morfill, Phys. Rev. E 66, 026411 (2002).

[26] P. K. Shukla, Phys. Plasmas 10, 1619 (2003); P. K. Shukla and A. A. Mamun, New J. Phys., 5, 17 (2003).

[27] K. Avinash, P. Zhu, V. Nosenko and J. Goree, Phys. Rev. E 68, 046402 (2003).

[28] M. Kong, B. Partoens and F. M. Peeters, New J. Phys. 5, 23.1 (2003); H. Totsuji, C. Totsuji and K. Tsuruta, Phys. Rev. E 64, 066402 (2001).

[29] J. M. Tamga and M. Remoissenet and J. Pouget, Phys. Rev. B 47, 14866 (1993); idem, Phys. Rev. Lett. 75, 357 (1995).

[30] I. Kourakis and P. K. Shukla, "Nonlinear theory of solitary waves associated with longitudinal particle motion in lattices: Application to longitudinal grain oscillations in a dust crystal", European Physical Journal D (in press).

[31] T. Taniuti and N. Yajima, J. Math. Phys. 10, 1369 (1969); N. Asano, T. Taniuti and N. Yajima, J. Math. Phys. 10, 2020 (1969).

[32] A. Hasegawa, Plasma Instabilities and Nonlinear Effects (Springer-Verlag, Berlin, 1975).

[33] A. C. Newell, Solitons in Mathematics and Physics (SIAM Publ., Philadelphia, 1985).

[34] V. E. Zakharov and A. B. Shabat, Sov. Phys. - JETP 37, 823 (1973);

[35] R. Fedele, H. Schamel and P. K. Shukla, Phys. Scripta T 98, 18 (2002); R. Fedele and H. Schamel, Eur. Phys. J. B 27 313 (2002).

[36] These expressions may be obtained from the ones in Ref. [35], by transforming the variables therein into our notation as: $\rho \rightarrow \rho^{2}, x \rightarrow \zeta, \quad s \rightarrow \tau, \quad \rho_{m} \rightarrow \rho_{0}^{2}, \alpha \rightarrow 2 P, q_{0} \rightarrow-2 P Q, \Delta \rightarrow L, E \rightarrow \Omega, V_{0} \rightarrow V$; also see Ref. [12].

[37] Thierry Dauxois and Michel Peyrard, Phys. Rev. Lett. 70, 3935 (1993); T. Bountis et al., Phys. Lett. A 268, 50 (2000); also see several papers in the volume: G. Tsironis and E. N. Economou (Eds.), Fluctuations, Disorder and Nonlinearity, Physica D 113, North-Holland, Amsterdam (1998).

[38] S.A.Kiselev, A. J. Sievers and S. Takeno, Phys. Rev. Lett. 61, 970 (1988); S.R.Bickham and A.J.Sievers, Comm. Cond. Mat. Phys. 17, 135 (1995).

[39] G. Morfill, A. V. Ivlev and J. R. Jokipii, Phys. Rev. Lett. 83 (5), 971 (2000); A. Ivlev, U. Konopka and G. Morfill, Phys. Rev. E 62, 2739 (2000); A. Ivlev and G. Morfill, Phys. Rev. E 63, 016409 (2000). 
Figure 1.

\section{Figure captions}

(a) The characteristic physical quantities defined in (8) - (10) (for Debye-type first neighbour interactions) are depicted against the lattice parameter $\kappa$ : the eigenfrequency (square) $\omega_{L, 0}^{2}$, normalized by $Q^{2} / M \lambda_{D}^{3}$ (solid curve -); the sound speed (square) $v_{0}^{2}$, normalized by $Q^{2} / M \lambda_{D}$ (long-dashed curve - - -); the cubic potential nonlinearity coefficient $p_{0}$, normalized by $Q^{2} / M \lambda_{D}$ (dot curve $\left.\cdots\right)$; the quartic potential nonlinearity coefficient $q_{0}$, normalized by $Q^{2} /\left(M \lambda_{D}\right)^{1 / 2} ;$ short-dashed curve - - ); (b) close-up near $\kappa \approx 1$.

Figure 2.

(a) The linear oscillation frequency $\omega$ (normalized byr $\left.\hat{\omega}_{0}=\left(2 Q^{2} / M \lambda_{D}^{3}\right)^{1 / 2}\right)$ is depicted against the normalized wavenumber $k r_{0}$; the three curves, from bottom to top, depict: the exact relation (11) (-), the continuum approximation (14) (---), and the (tangent) acoustic limit $\omega=k v_{0}(---)$; (b) close-up near the origin.

Figure 3.

The NLS coefficients $P, Q$ and their ratio $\xi=P / Q$ : discrete model [see (11)] (dashed curve) vs. continuum model (straight curve), are depicted vs. the (normalized) wavenumber $k r_{0}$, for $\kappa=1.25$. (a) $P$, cf. (19) (normalized by $\left(Q^{2} \lambda_{D} / M\right)^{1 / 2}$ ); (b) $Q$, cf. (24) (normalized by $\left[Q^{2} /\left(M \lambda_{D}^{7}\right)\right]^{1 / 2}$ ); (c) $\xi=P / Q$ (normalized by $\lambda_{D}^{4}$; divide by a factor $\kappa^{4}=1.25^{4} \approx 2.44$ to obtain the value in $\left.r_{0}^{4}\right)$.

Figure 4.

Similar to the previous figure, for $\kappa=0.25$. The continuum model seems to fail completely above $k=1$ : notice the discrete (correct) curve, which is hardly visible (dashed curve).

Figure 5.

(a) Bright (asymmetric) envelope solution $(P Q>0)$ : the first- and zeroth- harmonic components of the particle displacement, $u_{1}^{(1)}$ and $u_{0}^{(1)}$, are depicted, for $t=0$; dash $(---)$ and solid (-) curves, respectively. (b) The total displacement $u_{1}(\sim \epsilon)$ is depicted (arbitrary parameter values).

Figure 6.

Asymmetric envelope solutions for $P Q<0$ : the total displacement is depicted for $t=0$ (arbitrary parameter values): (a) grey-type envelope solution $(0<|a|<1)$; (a) dark-type envelope solution $(|a|=1)$.

Figure 7 .

Contours of the reduced ratio $\xi=P / Q$ (expressed in units $r_{0}^{4}$ ) are depicted vs. the (normalized) wavenumber $k r_{0}$ and the lattice parameter $\kappa$. (a) The $\xi$ values are, from top left and downwards: $-0.002,-0.005,-0.01,-0.02,-0.03$. The absolute value of the (negative) ratio increases with decreasing $\kappa$ and with increasing $k$. Notice the curly-shaped curve at the bottom, indicating the poles of the ratio $P / Q$, i.e. where $Q=0$ ( $\xi>0$ below this curve), as well as the resonance asymptotic line at $k r_{0}=3$. (b) Close up for small $\kappa$ and positive $\xi$ (wave instability region). The $\xi$ values are, from bottom to top: $0.35,0.4,0.5,1,2$ (and $+\infty$, again, i.e. $Q=0$, on top). 

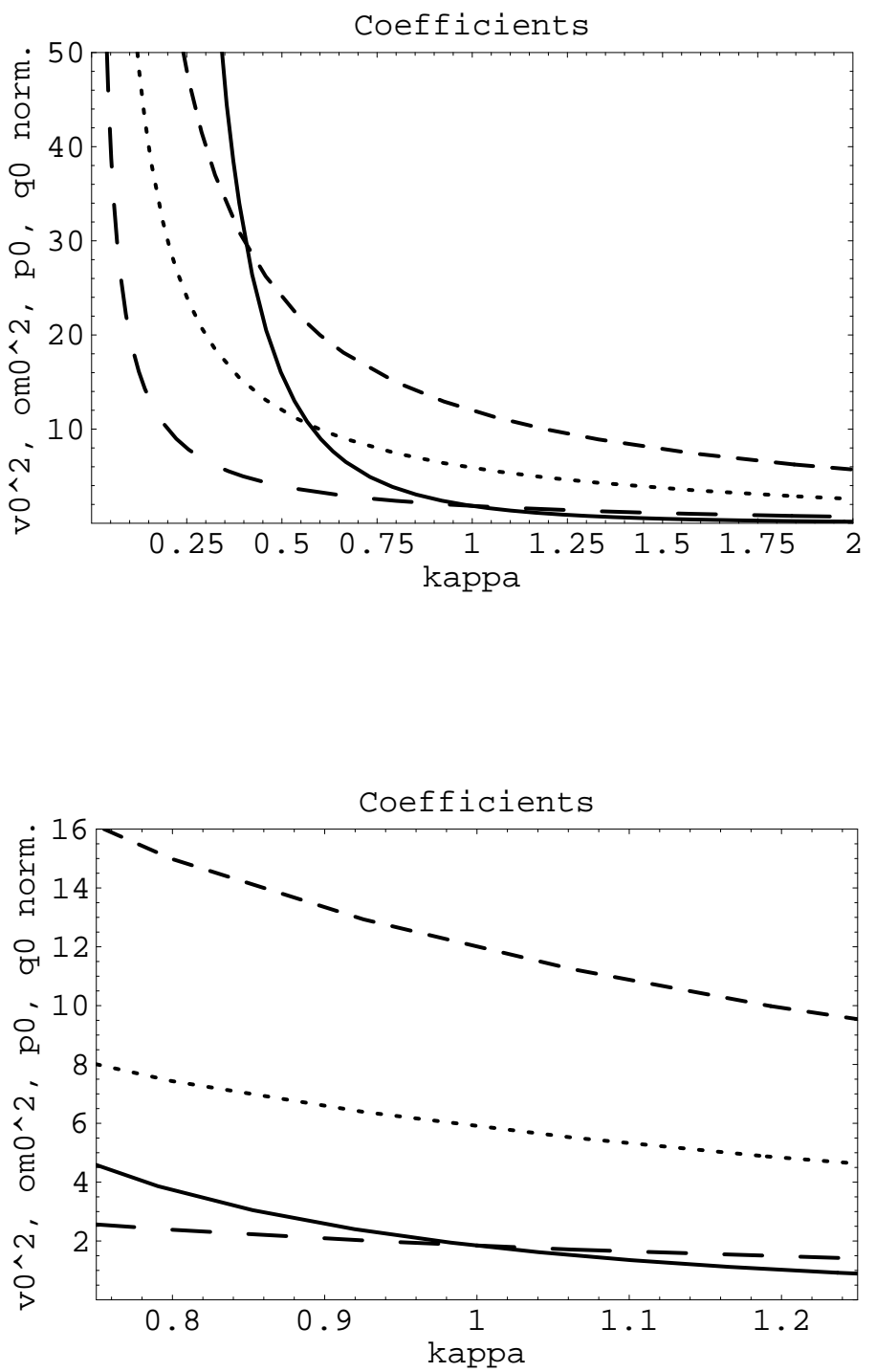

FIG. 1: 

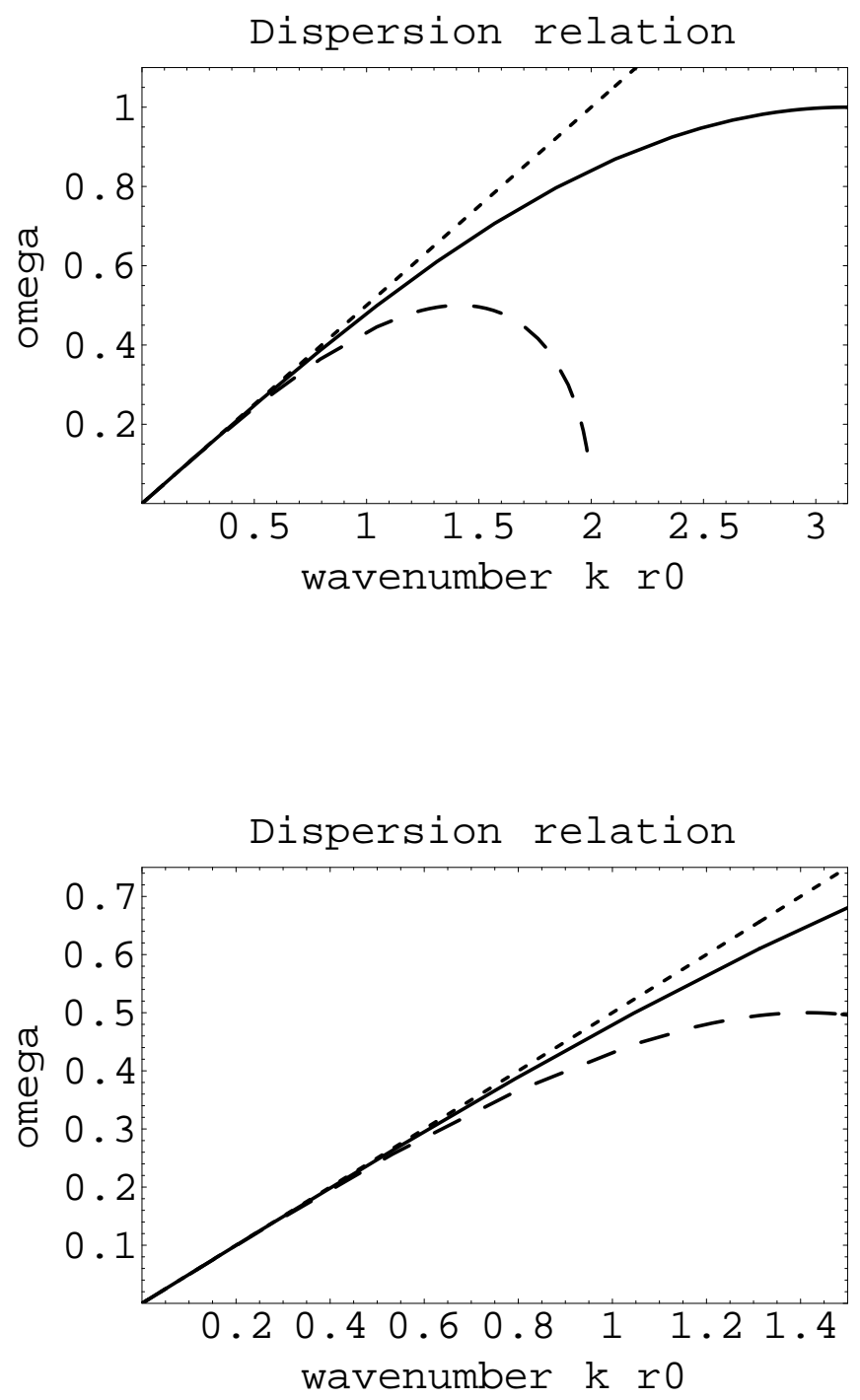

FIG. 2: 

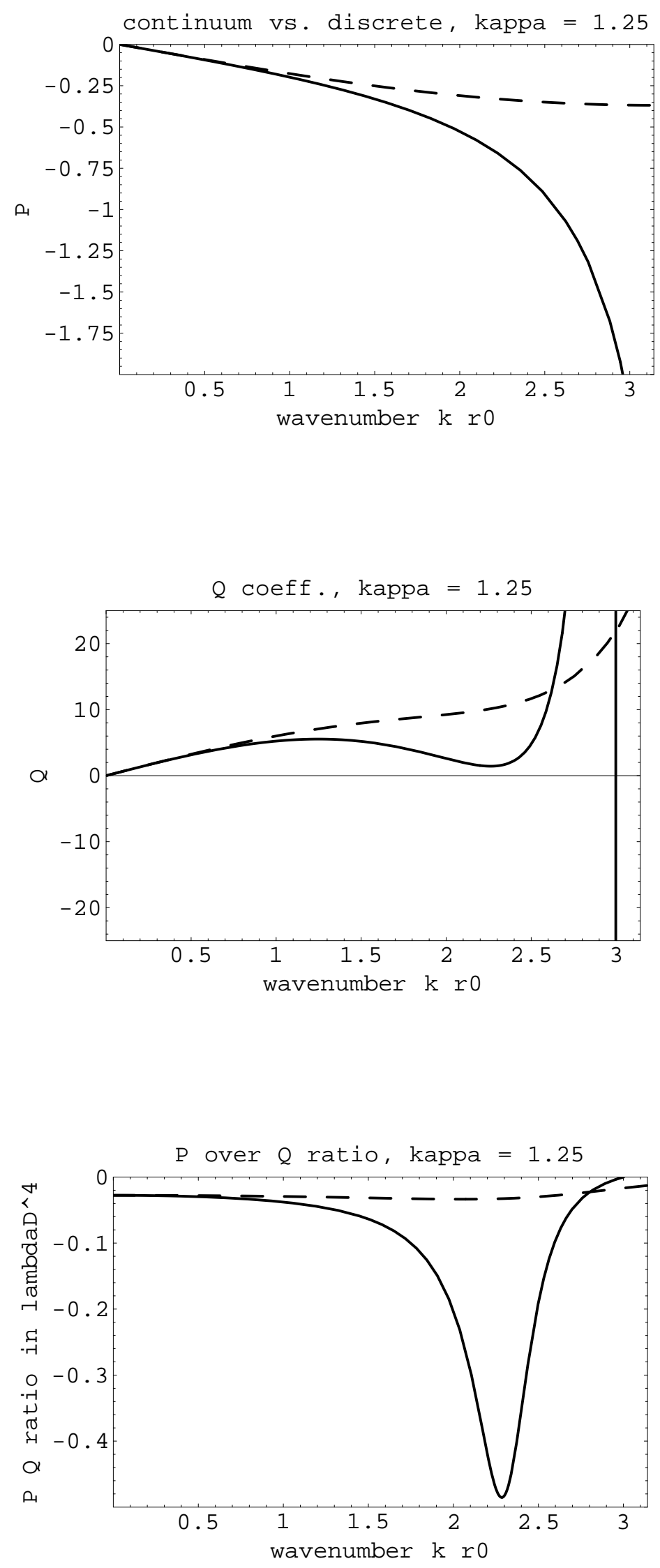

FIG. 3: 

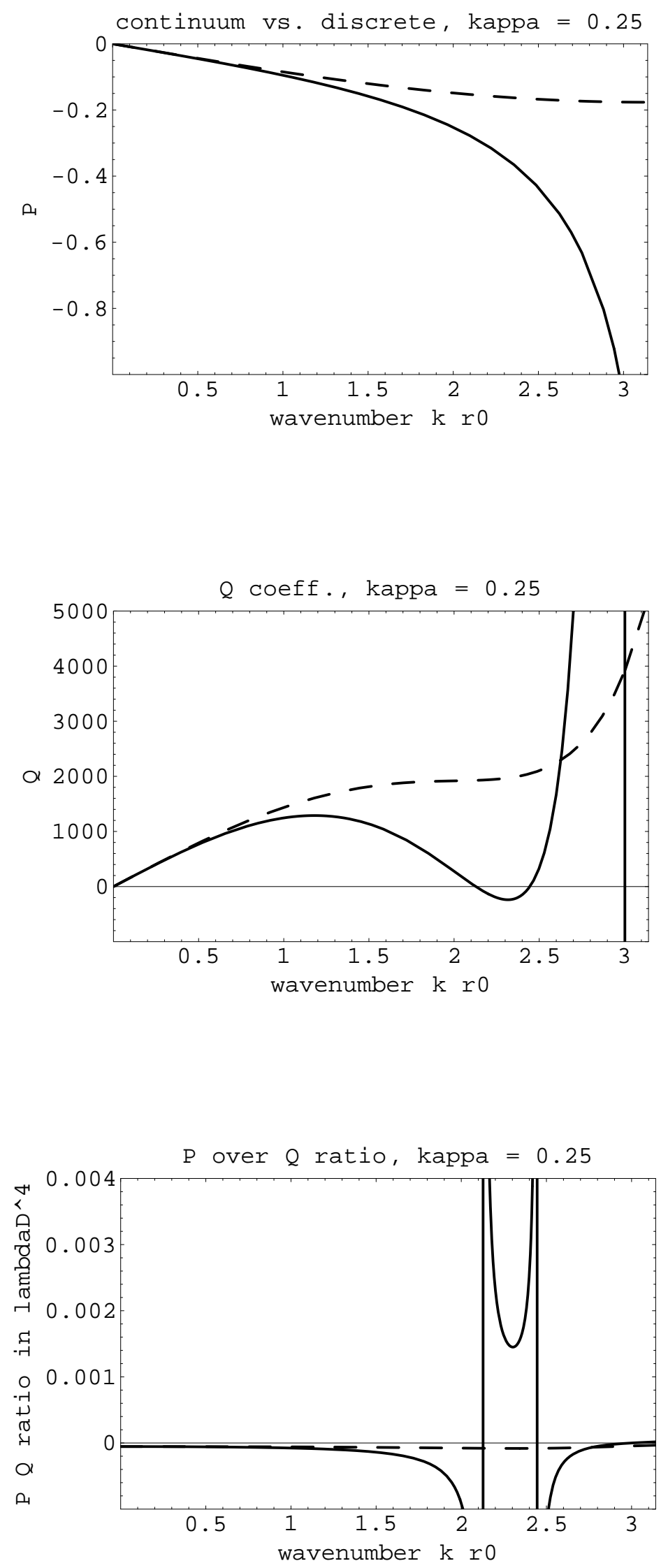

FIG. 4: 

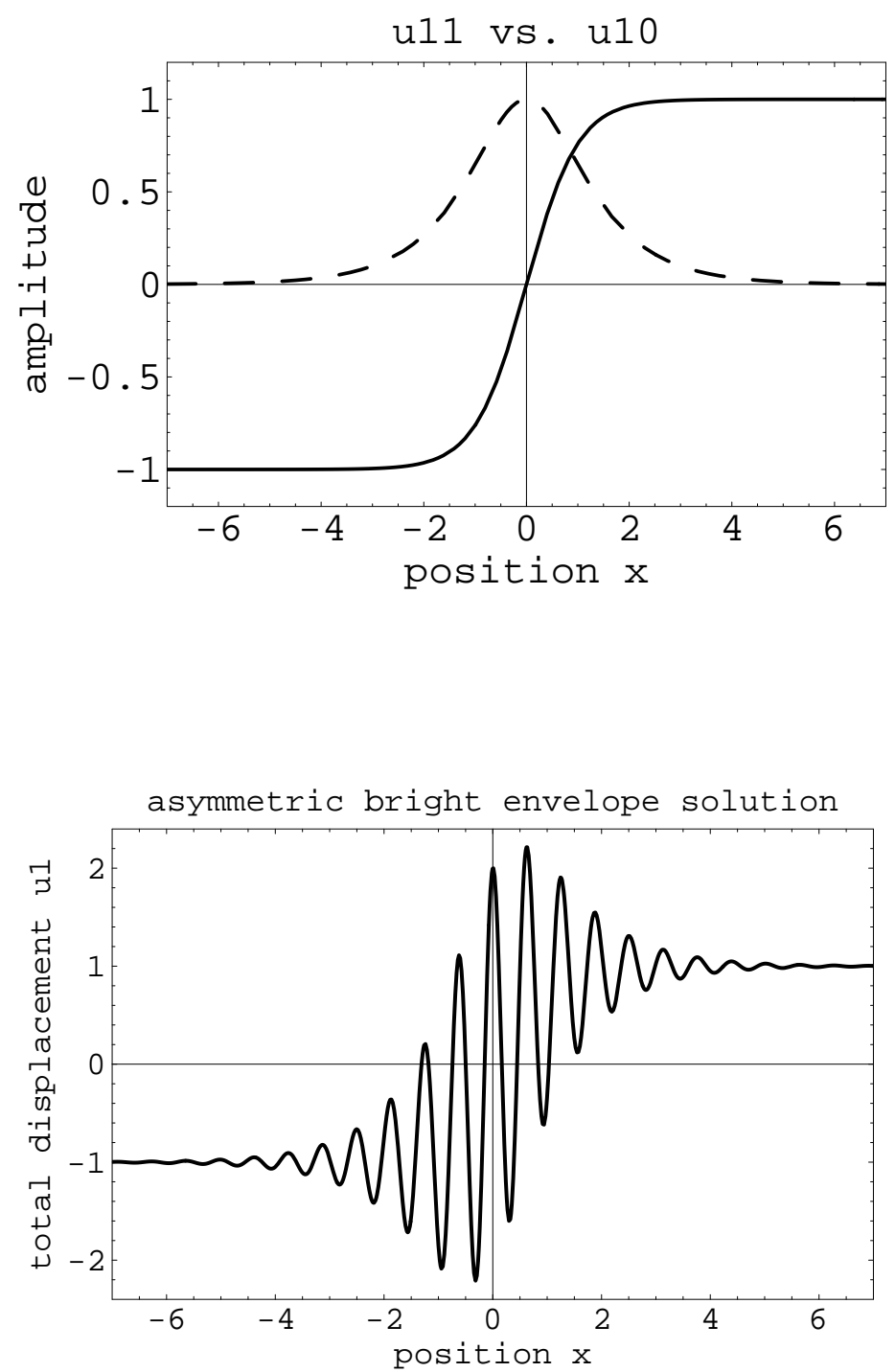

FIG. 5: 

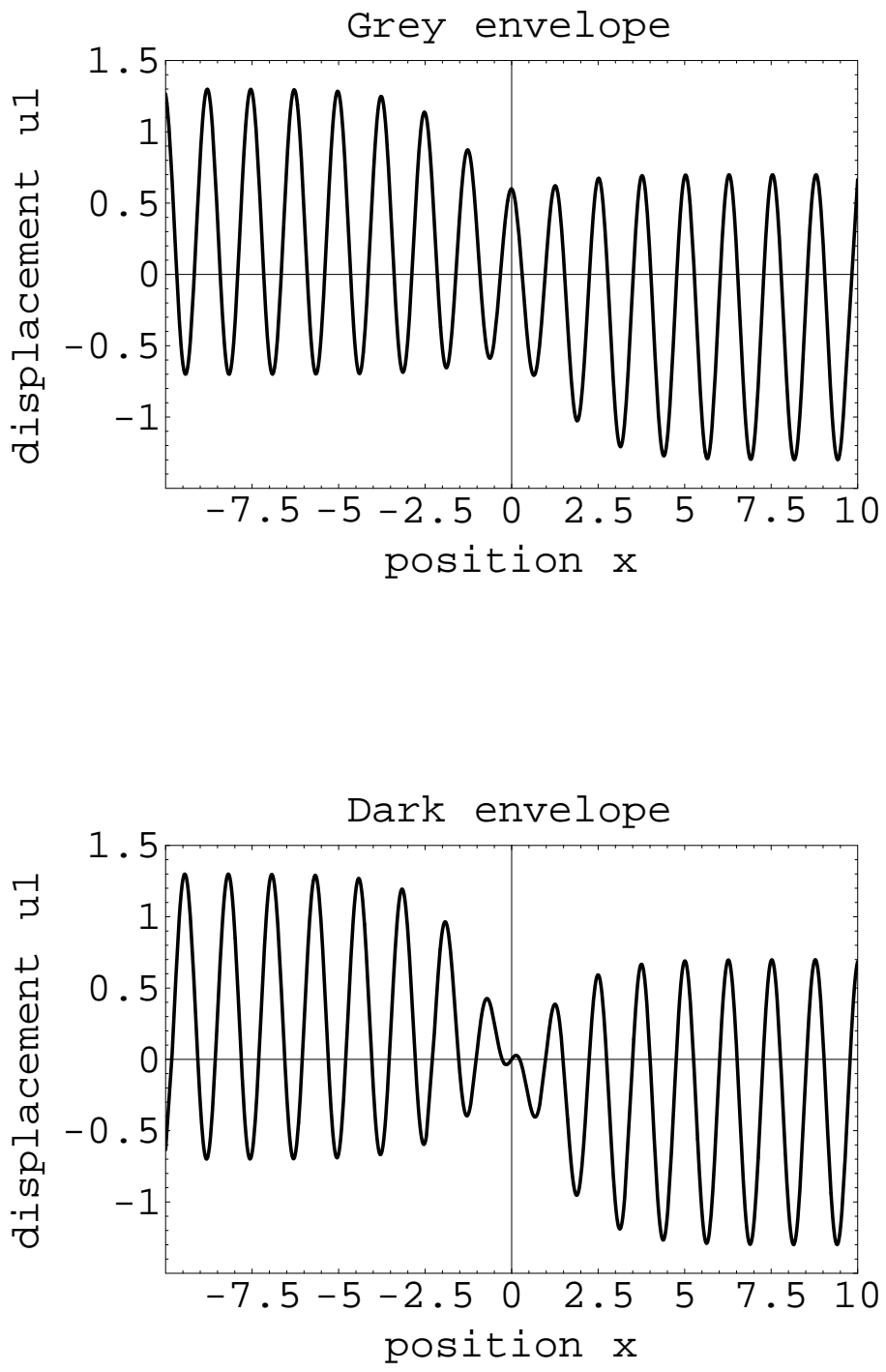

FIG. 6: 

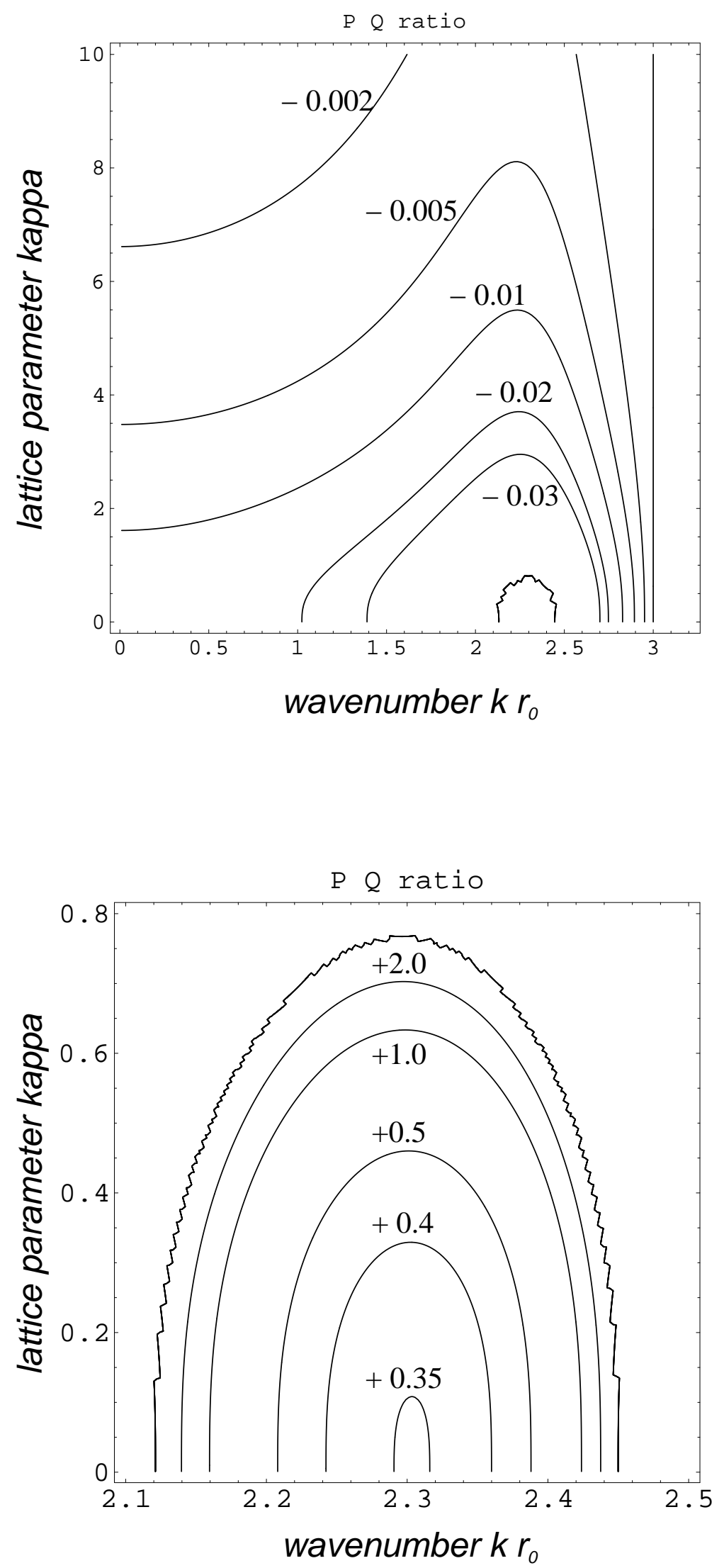

FIG. 7: 